\section{Primer estudio mexicano comparativo entre prostatectomía radical abierta y radical laparoscópica asistida por robot}

Herrera-Muñoz JA, Gómez-Sánchez J, Preciado-Estrella D, Trujillo-Ortiz L, Sedano-Basilio J, López-Maguey RP, Sánchez-Aquino U, Viana-Álvarez G, Veliz-Cabrera G, Cortes-Raygosa P, Ortega-González M, Calvo-Vázquez I, Hernández-Méndez E, Fernández-Noyola G, Cantellano-Orozco M, Martínez-Arroyo C, Morales-Montor JG, Pacheco-Gahbler C

\begin{abstract}
Resumen
ANTECEDENTES: hay controversia en cuanto a los resultados perioperatorios, oncológicos y funcionales entre la prostatectomía radical abierta versus prostatectomía radical laparoscópica asistida por robot.
\end{abstract}

OBJETIVO: comparar los resultados perioperatorios, oncológicos y funcionales entre la prostatectomía radical abierta versus prostatectomía radical laparoscópica asistida por robot.

MATERIALES Y MÉTODOS: estudio retrospectivo, descriptivo y analítico, efectuado mediante el análisis de los expedientes de pacientes posoperados de prostatectomía radical laparoscópica asistida por robot y prostatectomía radical abierta, de enero de 2012 a junio de 2016. Se analizaron variables preoperatorias (edad, estado funcional según los criterios del ECOG, comorbilidades, concentración de antígeno prostático específico, escala de Gleason y grupo de riesgo de la NCCN), perioperatorias (sangrando transoperatorio, tiempo quirúrgico, requerimiento de transfusión sanguínea, días de estancia hospitalaria, tiempo de permanencia de la sonda transuretral y complicaciones posquirúrgicas) y oncológicas (estadio clínico, criterios de Gleason de la pieza quirúrgica, márgenes positivos, persistencia y recurrencia bioquímica de la enfermedad; continencia urinaria [3, 6 y 12 meses] y potencia o desempeño sexual [6 y 12]). Para el análisis estadístico se utilizó el programa SPSS; se calcularon frecuencias y pruebas de normalidad. Se implementó la $\chi^{2}$ de Pearson para variables cualitativas, y t de Student y U de Mann-Whitney para variables cuantitativas.

RESULTADOS: se registraron 35 pacientes con prostatectomía radical laparoscópica asistida por robot y 36 con prostatectomía radical abierta. La edad media de los pacientes fue de 64 vs 63 años; tiempo quirúrgico de $286 \pm 68$ vs $225 \pm 65$ minutos $(p<0.001)$; sangrando transoperatorio de $869 \pm 657$ vs 1567 $1110 \mathrm{~mL}(p=0.001)$; tasa de transfusión de 37 vs $47 \%(p=0.26)$, respectivamente, y estancia hospitalaria de 5 días para ambos procedimientos. Se registraron complicaciones posquirúrgicas en 29 vs 28\% $(p=0.58)$ de los pacientes; márgenes positivos de 63 vs 33\% $(p=0.01)$ y tasa de continencia a los 3 meses de 57 vs $53 \%(p=0.7)$, respectivamente. El seguimiento de los pacientes con prostatectomía asistida por robot fue de 3 a 21 meses y de prostatectomía radical abierta de 1 a 4 años.

CONCLUSIONES: este estudio confirma las ventajas de la prostatectomía radical laparoscópica asistida por robot: menor sangrado tran-
División de Urología, Hospital General Dr. Manuel Gea González, SSA, Ciudad de México, México.

Recibido: diciembre 2016

Aceptado: abril 2017

\section{Correspondencia}

Javier Antonio Herrera Muñoz

dr.javierantonio.herrera@gmail.com

Este artículo debe citarse como

Herrera-Muñoz JA, Gómez-Sánchez J, PreciadoEstrella D, Trujillo-Ortiz L, Sedano-Basilio J, LópezMaguey RP, Sánchez-Aquino U, Viana-Álvarez G, Veliz-Cabrera G, Cortes-Raygosa P, Ortega-González M, Calvo-Vázquez I, Hernández-Méndez E, FernándezNoyola G, Cantellano-Orozco M, Martínez-Arroyo C, Morales-Montor JG, Pacheco-Gahbler C. Primer estudio mexicano comparativo entre radical abierta y prostatectomía radical laparoscópica asistida por robot. Rev Mex Urol. 2017 mayo;77(3):173-182. 
soperatorio y de trasfusión; los parámetros restantes fueron similares, pero no inferiores, a los de la prostatectomía radical abierta.

PALABRAS CLAVE: prostatectomía radical laparoscópica asistida por robot, prostatectomía radical abierta.

\section{First Mexican study comparing open radical prostatectomy and robotic-assisted laparoscopic radical prostatectomy}

Herrera-Muñoz JA, Gómez-Sánchez J, Preciado-Estrella D, Trujillo-Ortiz L, Sedano-Basilio J, López-Maguey RP, Sánchez-Aquino U, Viana-Álvarez G, Veliz-Cabrera G, Cortes-Raygosa $\mathrm{P}$, Ortega-González M, Calvo-Vázquez I, Hernández-Méndez E, Fernández-Noyola G, Cantellano-Orozco M, Martínez-Arroyo C, Morales-Montor JG, Pacheco-Gahbler C

\section{Abstract}

BACKGROUND: Perioperative, oncologic, and functional results between open radical prostatectomy and robotic-assisted laparoscopic radical prostatectomy are still the subject of debate.

OBJECTIVE: To compare the perioperative, oncologic, and functional results between open radical prostatectomy and robotic-assisted laparoscopic radical prostatectomy.

MATERIALS AND METHODS: A retrospective, descriptive, and analytic study was conducted by analyzing the medical records of patients that underwent robotic-assisted laparoscopic radical prostatectomy and patients that underwent open radical prostatectomy, within the time frame of January 2012 and June 2016. The follow-up period for the patients with robotic-assisted prostatectomy was from 3 to 21 months and that of open radical prostatectomy was from 1 to 4 years. Preoperative variables (age, ECOG functional status, comorbidities, prostate-specific antigen concentration, Gleason score, NCCN risk group), perioperative variables (intraoperative blood loss, surgery duration, days of hospital stay, time with transurethral catheter, postoperative complications), and oncologic variables (clinical stage, surgical specimen Gleason criteria, positive surgical margins, disease persistence and biochemical recurrence, urinary continence [at 3, 6, and 12 months], sexual potency or performance [at 6 and 12 months]) were analyzed. The SPSS program was used for the statistical analysis. Frequencies were calculated and normality tests were performed. The Pearson's $\chi^{2}$ test was employed for the qualitative variables and the Student's $t$ test and Mann-Whitney $U$ test were used for the quantitative variables. 
RESULTS: Thirty-five patients underwent robotic-assisted laparoscopic radical prostatectomy and 36 had open radical prostatectomy. For robotic-assisted laparoscopic radical prostatectomy versus open radical prostatectomy, the mean age of the patients was 64 vs 63 years, surgery duration was $286 \mathrm{~min}(68)$ vs $225 \mathrm{~min}( \pm 65)(\mathrm{p}<0.001)$. intraoperative blood loss was $869 \mathrm{~mL}( \pm 657)$ vs $1567 \mathrm{~mL}( \pm 1110)(\mathrm{p}=0.001)$, and the transfusion rate was 37 vs $47 \%(p=0.26)$, respectively. Mean hospital stay was 5 days for the two procedures. Postoperative complications presented in 29 vs $28 \%$ ( $p=0.58$ ) of the patients, there were positive surgical margins in 63 vs 33\% ( $=0.01)$, and the continence rate at 3 months was 57 vs 53\% ( $\mathrm{p}=0.7)$, respectively.

CONCLUSIONS: Our study confirmed the benefits of robotic-assisted laparoscopic radical prostatectomy in relation to intraoperative blood loss and transfusion. In addition, the rest of the parameters evaluated were similar, but not inferior, to those for open radical prostatectomy.

KEY WORDS: Robotic-assisted laparoscopic radical prostatectomy; Open radical prostatectomy
División de Urología, Hospital General Dr. Manuel Gea González, SSA, Ciudad de México, México.

Correspondence

Javier Antonio Herrera Muñoz dr.javierantonio.herrera@gmail.com

\section{ANTECEDENTES}

La prostatectomía radical abierta se describió en 1950, pero fue hasta 1980 cuando Walsh la popularizó; durante muchos años fue el patrón de referencia del tratamiento del cáncer de próstata. ${ }^{1}$ Por su parte, la prostatectomía radical laparoscópica asistida por robot la inició Binder en el año 2000; a partir de entonces se extendió en Estados Unidos. ${ }^{2}$ Hoy en día se calcula que entre 67 y $85 \%$ de las prostatectomías radicales son robóticas en ese país. ${ }^{3}$

Aunque los objetivos oncológicos y funcionales son los mismos para ambas opciones de tratamiento, existen diferencias en la técnica quirúrgica. La prostatectomía radical abierta, después de la ligadura del complejo venoso dorsal, implica la disección de la próstata, que se inicia en la apical al seccionar la uretra y se va lateral; este acceso difiere de la técnica robótica, donde después de la ligadura del complejo venoso dorsal, la disección se inicia con el cuello vesical y en la cara posterior de la próstata hacia las vesículas seminales. En ambas técnicas, siempre que sea factible, se procura la neuropreservación. La anastomosis descrita en la prostatectomía radical abierta consiste en aplicar puntos separados, del cuello hacia la uretra, lo que difiere con la prostatectomía radical laparoscópica asistida por robot, donde la sutura suele ser continua, con o sin puntos de Rocco. ${ }^{2,4}$

Las ventajas de la prostatectomía radical laparoscópica asistida por robot versus radical abierta aún se discuten. Diversas series refieren menor sangrado transoperatorio, tasa de transfusión y estancia hospitalaria, aunque sin diferencias en otras complicaciones posquirúrgicas. ${ }^{1,5}$ Los estudios recientes reportan menor morbilidad perioperatoria y mejor control oncológico cuando se practica la prostatectomía radical laparoscópica asistida por robot; sin embargo, su costo es muy elavado. ${ }^{6-8}$ Por lo que se refiere a los resultados funcionales, ha demostrado 
mejor recuperación en la continencia urinaria y el desempeño sexual. ${ }^{9,10}$

En México, pocos hospitales tienen la posibilidad de contar con el sistema Da Vinci para efectuar la prostatectomía radical laparoscópica asistida por robot. En la División de Urología del Hospital General Dr. Manuel Gea González se ha iniciado la curva de aprendizaje con esta técnica que, a diferencia de la prostatectomía radical abierta, se han Ilevado a cabo más de 200 procedimientos; por lo tanto, es importante realizar una primera comparación entre ambos abordajes, con la finalidad de conocer los resultados perioperatorios, oncológicos y funcionales.

\section{MATERIALES Y MÉTODOS}

Estudio retrospectivo, descriptivo y analítico. Se reunieron los expedientes de pacientes posoperados de prostatectomía radical laparoscópica asistida por robot y prostatectomía radical abierta de enero de 2012 a junio de 2016.

Se analizaron las variables preoperatorias: edad, estado funcional según los criterios del Eastern Cooperative Oncology Group (ECOG), comorbilidades, concentración de antígeno prostático específico (APE), escala de Gleason y grupo de riesgo de la National Comprehensive Cancer Network (NCCN). Las variables perioperatorias analizadas fueron: sangrando transoperatorio, tiempo quirúrgico, requerimiento de transfusión sanguínea, días de estancia hospitalaria, tiempo de permanencia de la sonda transuretral y complicaciones posquirúrgicas en los primeros 90 días relacionadas con la intervención quirúrgica.

Las variables oncológicas analizadas fueron: estadio clínico, criterios de Gleason de la pieza quirúrgica, márgenes positivos, persistencia y recurrencia bioquímica de la enfermedad, y necesidad de radioterapia coadyuvante. También se evaluaron: la continencia urinaria a los 3, 6 y 12 meses y la potencia o desempeño sexual a los 6 y 12 meses con ambos procedimientos.

Se definió margen positivo cuando se identificaron células cancerígenas en la superficie con tinción del espécimen y se dividieron según su localización, sobre todo del ápex. ${ }^{11}$ La recurrencia bioquímica se definió como dos elevaciones del antígeno prostático específico mayores de $0.2 \mathrm{ng} / \mathrm{mL}$ después de la prostatectomía radical y concentración posoperatorias inicial de $0 \mathrm{ng} /$ $\mathrm{mL}$. Se consideró persistencia de la enfermedad a la concentración de antígeno prostático específico mayor de $0.2 \mathrm{ng} / \mathrm{mL}$ luego de la primera determinación posquirúrgica. ${ }^{12}$

La continencia urinaria se definió como la no utilización de toalla absorbente y ausencia de fuga durante la aplicación de esfuerzo físico. ${ }^{9}$ Se consideró función sexual adecuada cuando el paciente pudo mantener el coito en más de $50 \%$ de las ocasiones, con o sin prescripción de inhibidores de 5 -fosfodiesterasa o un IIFE de 17 o mayor. ${ }^{13,14}$

Para el análisis estadístico se utilizó el programa SPSS; se calcularon frecuencias y pruebas de normalidad. Se implementó la $\chi^{2}$ de Pearson para variables cualitativas, y la t de Student y $U$ de Mann-Whitney para variables cuantitativas.

\section{RESULTADOS}

Durante el periodo de estudio se realizaron 36 prostatectomías radicales abiertas y 35 prostatectomías laparoscópicas asistidas por robot, con seguimiento de 1 a 4 años versus 3 a 21 meses, respectivamente. La edad media de los pacientes tratados mediante prostatectomía radical abierta fue de 63 años (47-74) y de prostatectomía robótica de 64 años (45-76). De acuerdo con los criterios del ECOG, el puntaje del estado funcional fue 0 en $98 \%$ de los casos y todos mostraron continencia preoperatoria. Se registra- 
ron comorbilidades en 18 (50\%) pacientes con prostatectomía radical abierta y en 15 (42.9\%) de prostatectomía laparoscópica robótica, sin observarse diferencias significativas $(p=0.45)$; en ambos grupos la hipertensión arterial fue la más común (Cuadro 1). La concentración media prequirúrgica de antígeno prostático específico fue similar en ambos grupos ( $p=0.49$ ). En cuanto al puntaje de Gleason prequirúrgico de pacientes con prostatectomía radical abierta y prostatectomía radical laparoscópica asistida por robot, el más frecuente fue $3+3=6$ en 83.3 y $65.7 \%$, respectivamente. De acuerdo con los grupos de riesgo de la $\mathrm{NCCN}$, ninguno reportó diferencias significativas ( $p=0.46$ ); sin embargo, el grupo de riesgo con mayor prevalencia fue el intermedio en $38.9 \%$ para prostatectomía radical abierta y $45.7 \%$ para laparoscopia asistida por robot (Cuadro 1).
En el análisis de las variables perioperatorias (Cuadro 2), el tiempo quirúrgico promedio para prostatectomía radical abierta fue de 225 minutos $( \pm 65.3)$ y para prostatectomía radical laparoscópica asistida por robot de 286 minutos $( \pm 67.9)(p<0.01)$. El sangrando transoperatorio medio de la prostatectomía radical abierta fue de $1,567 \mathrm{~mL}$ ( 1110) y laparoscopia robótica de 869 $\mathrm{mL}( \pm 657)$ ( $p=0.001)$. La tasa de transfusión fue menor en pacientes con prostatectomía radical laparoscópica asistida por robot versus prostatectomía radical abierta (37.1 vs $47.2 \%$ ), pero sin significación estadística $(\mathrm{p}=0.26)$.

Se registraron complicaciones posquirúrgicas en $28 \%$ de los pacientes con prostatectomía radical abierta y en $29 \%$ de prostatectomía robótica $(\mathrm{p}=0.94)$. En los primeros, $3(8.3 \%)$ padecieron íleo, 2 (5.6\%) tuvieron hematuria

Cuadro 1. Características preoperatorias de ambas técnicas quirúrgicas

\begin{tabular}{|c|c|c|c|}
\hline Variable & $\begin{array}{c}\text { PRA } \\
(\mathrm{n}=36)\end{array}$ & $\begin{array}{c}\text { Prostatectomía radical laparoscópica } \\
\text { asistida por robot }(\mathrm{n}=35)\end{array}$ & $\stackrel{p}{\text { IC }} \stackrel{95 \%}{2}$ \\
\hline \multicolumn{4}{|c|}{$\mathrm{DE}$} \\
\hline $\begin{array}{l}\text { Edad (años) } \\
\text { APE prequirúrgico } \\
(\mathrm{ng} / \mathrm{mL})\end{array}$ & $\begin{array}{c}63 \pm 7 \\
10.8 \pm 62\end{array}$ & $\begin{array}{c}64 \pm 7 \\
9.3 \pm 3.9\end{array}$ & $\begin{array}{l}0.88 \\
0.49\end{array}$ \\
\hline \multicolumn{4}{|c|}{ n (\%) } \\
\hline $\begin{array}{l}\text { Comorbilidades } \\
\text { Hipertensión } \\
\text { Diabetes } \\
\text { Hipotiroidismo } \\
\text { Artritis reumatoide } \\
\text { Insuficiencia cardiaca } \\
\text { RTUP previa }\end{array}$ & $\begin{array}{c}18(50) \\
10(27.8) \\
4(11.1) \\
- \\
- \\
1(2.8) \\
4(11.1)\end{array}$ & $\begin{array}{c}15(42.9) \\
11(31.4) \\
4(11.1) \\
1(2.9) \\
1(2.9) \\
- \\
-\end{array}$ & 0.45 \\
\hline Gleason prequirúrgico & & & 0.39 \\
\hline $\begin{array}{l}3+3=6 \\
3+4=7 \\
4+3=7 \\
4+4=8 \\
4+5=9\end{array}$ & $\begin{array}{c}30(83.3) \\
3(8.3) \\
2(5.6) \\
1(2.8) \\
-\end{array}$ & $\begin{array}{c}23(65.7) \\
5(14.3) \\
4(11.4) \\
1(2.9) \\
2(5.7)\end{array}$ & \\
\hline $\begin{array}{l}\text { Grupo de riesgo } \\
\text { Muy bajo } \\
\text { Bajo } \\
\text { Intermedio } \\
\text { Alto }\end{array}$ & $\begin{array}{c}8(22.2) \\
11(30.6) \\
14(38.9) \\
3(8.3)\end{array}$ & $\begin{array}{c}3(8.3) \\
12(34.3) \\
16(45.7) \\
4(11.4)\end{array}$ & 0.46 \\
\hline
\end{tabular}

PRA: prostatectomía radical abierta; APE: antígeno prostático específico; RTUP: resección transuretal de próstata; IC: intervalo de confianza; DE: desviación estándar. 
Cuadro 2. Resultados perioperatorios

\begin{tabular}{|c|c|c|c|}
\hline Variable & $\begin{array}{c}\text { PRA } \\
(n=36)\end{array}$ & $\begin{array}{c}\text { Prostatectomía } \\
\text { radical } \\
\text { laparoscópica } \\
\text { asistida } \\
\text { por robot }(n=35)\end{array}$ & 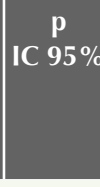 \\
\hline & $\mathrm{DE}$ & & \\
\hline $\begin{array}{l}\text { Tiempo } \\
\text { quirúrgico } \\
\text { (minutos) }\end{array}$ & $\begin{array}{l}225 \\
65.3\end{array}$ & $286 \pm 68$ & $<0.001$ \\
\hline $\begin{array}{l}\text { Sangrado } \\
\text { transoperatorio } \\
(\mathrm{mL})\end{array}$ & $\begin{array}{l}1567 \\
1110\end{array}$ & $869 \pm 657$ & 0.001 \\
\hline $\begin{array}{l}\text { Tiempo de sonda } \\
\text { transuretral } \\
\text { (días) }\end{array}$ & $\begin{array}{c}20 \pm \\
4.2\end{array}$ & $21 \pm 9.2$ & 0.31 \\
\hline \multirow{2}{*}{$\begin{array}{l}\text { Estancia } \\
\text { intrahospitalaria } \\
\text { (días) }\end{array}$} & $5 \pm 1.7$ & $5 \pm 4$ & 0.65 \\
\hline & n $(\%)$ & & \\
\hline Transfusión & $\begin{array}{c}17 \\
(47.2)\end{array}$ & $13(37.2)$ & 0.26 \\
\hline $\begin{array}{l}\text { Complicación } \\
\text { Clavien I } \\
\text { Clavien II } \\
\text { Clavien III } \\
\text { Clavien IV } \\
\text { Clavien V }\end{array}$ & $\begin{array}{c}10(28) \\
4 \\
(11.1) \\
3(8.3) \\
2(5.5) \\
1(2.7) \\
-\end{array}$ & $\begin{array}{c}10(29) \\
4(11.4) \\
4(11.4) \\
2(5.7) \\
1(2.9) \\
-\end{array}$ & 0.94 \\
\hline
\end{tabular}

PRA: prostatectomía radical abierta; DE: desviación estándar.

posquirúrgica, 2 (5.6\%) dehiscencia de anastomosis que ameritaron reintervención por cistoscopia, $1(2.7 \%)$ hematoma de la herida, $1(2.7 \%)$ pielonefritis y $1(2.7 \%)$ padeció una complicación cardiovascular que requirió su traslado a la unidad de cuidados intensivos; mientras que en los segundos, 3 (8.5\%) manifestaron infección urinaria (que requirió tratamiento intravenoso hospitalario), 3 (8.5\%) íleo, 1 (2.9\%) trombosis venosa profunda, 1 (2.9\%) neuropatía, 1 hematoma residual que se drenó por vía percutánea y 1 (2.9\%) con hemorragia posquirúrgica que requirió laparotomía exploradora e ingreso a la unidad de cuidados intensivos. En cuanto a la clasificación de Clavien, ninguna opción de tratamiento mostró diferencias en la clasificación-estadio III y IV (Cuadro 2). Ningún paciente de prostatectomía radical laparoscópica asistida requirió la conversión a procedimiento abierto, ni se registraron defunciones con una u otra opción (Clavien V).

Por lo que se refiere a los resultados oncológicos, el puntaje Gleason de la pieza más común fue de $3+3=6$ en $24(66.7 \%)$ pacientes con prostatectomía radical abierta y en 18 (51.4\%) con laparoscopia asistida por robot. Aunque el estadio más frecuente en ambos abordajes fue pT2c, la prostatectomía radical laparoscópica asistida por robot se asoció con estadios $\geq \mathrm{pT} 3$ $(\mathrm{p}=0.004)$; además, sólo el grupo de prostatectomía radical abierta reportó 3 casos con ganglios positivos (Cuadro 3).

La tasa de márgenes positivos fue menor en pacientes con prostatectomía radical abierta (33\%) respecto de los intervenidos mediante laparoscopia asistida por robot $(63 \%)(p=0.01)$. En ambos accesos el sitio más común de márgenes positivos fue el ápex. Aunque se registró mayor persistencia de enfermedad en 5 (14.2\%) pacientes con prostatectomía radical laparoscópica asistida por robot y en $3(8.3 \%)$ con prostatectomía radical abierta, no se observaron diferencias estadísticamente significativas $(p=0.42)$. Tampoco se registraron diferencias en la recurrencia bioquímica entre una y otra opción de tratamiento; sin embargo, debe considerarse un seguimiento más largo en los pacientes con prostatectomía radical laparoscópica asistida por robot para realizar una comparación más confiable entre ambos tratamientos. Todos los pacientes con persistencia y recurrencia bioquímica de enfermedad iniciaron tratamiento de pérdida androgénica y radioterapia. Al excluir estos pacientes, no se observaron diferencias significativas en quienes requirieron radioterapia coadyuvante por factores de mal pronóstico (Cuadro 3). 
Cuadro 3. Resultados oncológicos y funcionales
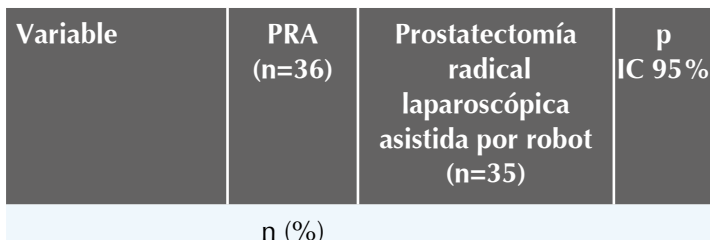

n $(\%)$

$\begin{array}{lcc}\begin{array}{l}\text { Estadio } \\ \text { patológico }\end{array} & & \\ \text { pT2a } & 11(30.6) & 3(8.6) \\ \text { pT2b } & 4(11.1) & - \\ \text { pT2c } & 13(36.1) & 18(51.4) \\ \text { pT3a } & 3(8.3) & 11(31.4) \\ \text { pT3b } & 2(5.6) & 3(8.6) \\ \text { N1 } & 3(4.2) & -\end{array}$

Gleason de la pieza quirúrgica

$3+3=6$

$3+4=7$

$24(66.7)$

$18(51.4)$

0.61

$4+3=7$

$10(27.8)$

$11(31.4)$

$1(2.8)$

$2(5.7)$

$4+4=8$

$3+5=8$

$4+5=9$

$5+5=10$

$1(2.8)$

$1(2.9)$

$2(5.7)$

$1(2.9)$

\begin{tabular}{|c|c|c|c|}
\hline $\begin{array}{l}\text { Márgenes } \\
\text { positivos }\end{array}$ & $12(33.3)$ & $22(63)$ & 0.01 \\
\hline $\begin{array}{l}\text { Persistencia de } \\
\text { la enfermedad }\end{array}$ & $3(8.3)$ & $5(14.3)$ & 0.42 \\
\hline $\begin{array}{l}\text { Recurrencia } \\
\text { bioquímica }\end{array}$ & $3(8.3)$ & $2(5.7)$ & 0.6 \\
\hline $\begin{array}{l}\text { Radioterapia } \\
\text { coadyuvante } \\
\text { (exclusión de } \\
\mathrm{N}+\text { y con } \\
\text { persistencia de } \\
\text { la enfermedad) }\end{array}$ & $6(16.7)$ & $6(17.1)$ & 0.9 \\
\hline \multicolumn{4}{|l|}{$\begin{array}{l}\text { Continencia } \\
\text { urinaria }\end{array}$} \\
\hline 3 meses & $19(53)$ & $20(57)$ & 0.7 \\
\hline 6 meses & 32 (88) & 19/25 (76) & 0.18 \\
\hline 12 meses & $34(94)$ & $14 / 14(100)$ & 0.36 \\
\hline \multicolumn{4}{|l|}{$\begin{array}{l}\text { Desempeño } \\
\text { sexual }\end{array}$} \\
\hline 3 meses & $2(5.6)$ & $1(2.9)$ & 0.5 \\
\hline 6 meses & $6(16.6)$ & $3 / 25$ & 0.61 \\
\hline 12 meses & $13(36)$ & $8 / 14(57)$ & 0.17 \\
\hline
\end{tabular}

PRA: prostatectomía radical abierta.
Por lo que se refiere a los resultados funcionales, la continencia urinaria (Figura 1) a los 3 meses de pacientes con prostatectomía radical laparoscópica asistida por robot fue de $57 \%$ (20 pacientes) y de prostatectomía radical abierta de $53 \%$ (19 pacientes) $(\mathrm{p}=0.7)$; a los 6 meses de $76 \%$ (19 de 25 pacientes evaluados) vs $88.9 \%$ (32 pacientes) $(\mathrm{p}=0.18)$ y a los 12 meses de $100 \%$ (14 de 14 pacientes evaluados) vs $94 \%$ (34 pacientes) $(\mathrm{p}=0.36)$, respectivamente; no hubo diferencia estadísticamente significativa entre ambas opciones de tratamiento.

La potencia o desempeño sexual (Figura 2) a los 3 meses en pacientes con prostatectomía radical laparoscópica asistida por robot fue de $2.9 \%$ ( 1 paciente) y con prostatectomía radical abierta de $5.6 \%$ ( 2 pacientes $)(p=0.5)$; a los 6 meses de $12 \%$ (3 de 25 pacientes evaluados) vs $16.6 \%$ ( 6 pacientes) ( $\mathrm{p}=0.61)$ y a los 12 meses de $57 \%$ (8 de 14 pacientes evaluados) vs 36\% (13 pacientes), respectivamente, sin observarse diferencias estadísticamente significativas $(p=0.17)$.

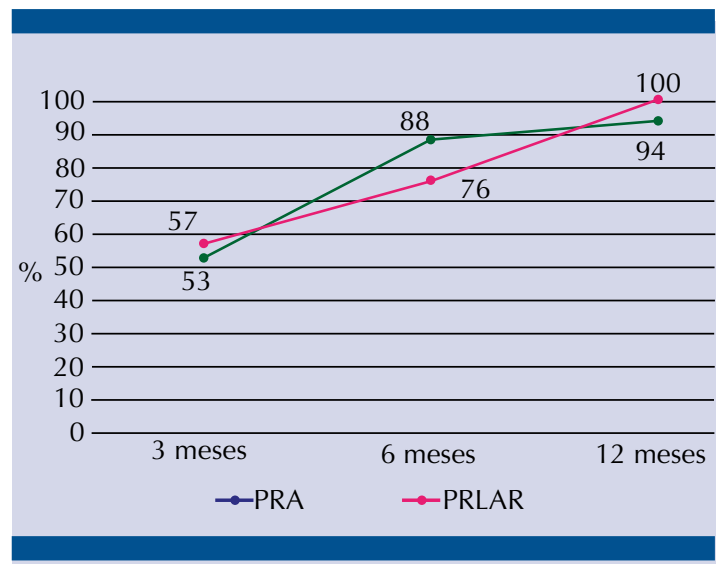

Figura 1. Relación entre ambos tratamientos quirúrgicos y tiempo para alcanzar la continencia urinaria. PRA: prostatectomía radical abierta; PRLAR: prostatectomía radical laparoscópica asistida por robot. 


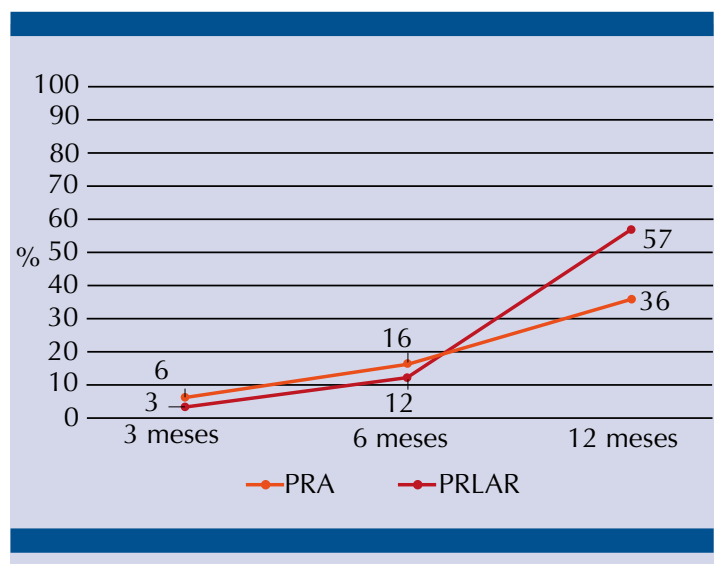

Figura 2. Relación entre ambos tratamientos quirúrgicos y potencia o desempeño sexual.

PRA: prostatectomía radical abierta; PRLAR: prostatectomía radical laparoscópica asistida por robot.

\section{DISCUSIÓN}

Los resultados perioperatorios, oncológicos y funcionales entre prostatectomía radical abierta y prostatectomía radical laparoscópica asistida por robot varían según la bibliografía internacional. En el estudio aquí reportado, ambos grupos tuvieron características preoperatorias similares. Gandaglia y su grupo fueron los primeros en reportar menor tasa de transfusión y estancia hospitalaria en pacientes tratados mediante prostatectomía radical laparoscópica asistida por robot. ${ }^{1}$ El metanálisis de Novara y colaboradores reportó baja tasa de sangrado (promedio de 582 $\mathrm{mL}$ ) y de transfusión en pacientes con prostatectomía radical laparoscópica asistida por robot. ${ }^{5}$ En nuestro estudio, la tasa de sangrado fue menor en el grupo de prostatectomía laparoscópica asistida por robot $(698 \mathrm{~mL})$ que el de prostatectomía radical abierta $(p<0.001)$, lo que coincide con lo reportado en la bibliografía. El requerimiento de trasfusión también fue menor en el mismo grupo (37.2 vs $47.2 \%$, respectivamente; $\mathrm{p}=0.26$ ), aunque sin diferencia significativa. Respecto al tiempo quirúrgico, Novara y sus coautores reportaron 15 minutos más al realizar prostatectomía radical laparoscópica asistida por robot versus radical abierta, sin diferencia significativa ( $p=0.56) .{ }^{5}$ Este dato difiere de nuestro estudio, donde la prostatectomía robótica tuvo duración significativamente mayor en 61 minutos $(p=0.001)$, similar a lo reportado por Jackson y colaboradores, con 56 minutos de diferencia entre ambos abordajes $(p<0.001),{ }^{15} y$ Leow $y$ su grupo, quienes reportan una diferencia de 131 minutos mayor con la misma técnica quirúrgica $(p=0.002){ }^{6}$

En cuanto a las complicaciones, Novara y su grupo, y Gandaglia y sus colaboradores no encontraron diferencias entre ambos procedimientos; sin embargo, Leow y sus coautores señalan que las complicaciones en los primeros 90 días del postoperatorio son $32 \%$ menores en pacientes con prostatectomía radical laparoscópica asistida por robot $(\mathrm{p}<0.001) ;{ }^{6}$ lo que difiere de nuestro estudio, donde las complicaciones fueron similares (28 vs 29\%, respectivamente; $\mathrm{p}=0.94$ ) entre uno y otro. Gandaglia y su grupo reportaron que la prostatectomía radical laparoscópica asistida por robot suele asociarse con complicaciones genitourinarias, lo que coincide con nuestros resultados, pues observamos que las infecciones urinarias fueron frecuentes con esta opción. ${ }^{1}$ La estancia hospitalaria reportada por Leow y colaboradores fue de 1.71 días en sujetos con prostatectomía radical laparoscópica asistida por robot versus 2.95 días de prostatectomía radical abierta $(\mathrm{p}<0.001),{ }^{6}$ dato diferente a nuestros resultados, que no muestran diferencias en la estancia hospitalaria, pues con ambas opciones se reportaron, en promedio, 5 días.

Otro metanálisis llevado a cabo por Novara y su grupo no mostró diferencias en los márgenes positivos entre prostatectomía radical abierta y prostatectomía robótica (21 vs $20 \%$; $\mathrm{p}=0.19) .{ }^{11}$ Recientemente, Pearce y sus colaboradores ${ }^{8}$ reportaron $2.3 \%$ menor tasa de márgenes positivos en pacientes con prostatectomía radical laparoscópica asistida por robot, al igual que Hu 
y su grupo ${ }^{7}$ (prostatectomía radical laparoscópica asistida por robot y prostatectomía radical abierta: 13.6 vs $18.3 \%$, respectivamente; $p<0.001$ ). Estos resultados difieren de los nuestros, donde se encontró menor tasa de márgenes positivos en la prostatectomía radical abierta versus robótica (33 vs 63\%; $p=0.01$ ), quizá porque se encontraron estadios patológicos $\geq \mathrm{pT} 3$ más frecuentes en pacientes con prostatectomía laparoscópica por robot versus prostatectomía radical abierta (40 vs $13.9 \% ; \mathrm{p}=0.004)$. Respecto de la persistencia de la enfermedad, Jackson y sus coautores no reportan diferencia entre uno y otro procedimiento $(\mathrm{p}=0.93) .{ }^{15}$ El metanálisis de Novara tampoco señala diferencias en la recurrencia bioquímica a 5 y 7 años $(p=0.14),{ }^{11}$ lo que coincide con nuestros resultados, donde la persistencia y recurrencia bioquímica fueron similares (8.3 vs 14.3\%; $\mathrm{p}=0.42$ para persistencia de enfermedad y 8.3 vs $5.7 \% ; \mathrm{p}=0.6$ para recurrencia bioquímica); $\sin$ embargo, es necesario aclarar que la recurrencia bioquímica en los pacientes de prostatectomía radical laparoscópica asistida por robot de nuestro estudio es poco valorable por el tiempo de seguimiento relativamente corto. En cuanto a la radioterapia coadyuvante, Hu y su grupo encontraron diferencias entre la prostatectomía radical abierta y la laparoscópica asistida por robot $(6.2$ vs $4.5 \%$; $\mathrm{p}<0.001),{ }^{7}$ al igual que Pearce y sus colaboradores ( 4.6 vs $2.6 \% ; p<0.01$ ), lo que difiere de nuestros resultados, que no mostraron diferencia significativa (16.7 vs $17.1 \% ; \mathrm{p}=0.9$ ).

En relación con los resultados funcionales, Ficarra y colaboradores señalan que la continencia urinaria a los 12 meses es mejor en pacientes con prostatectomía radical laparoscópica asistida $(p=0.03) .{ }^{9}$ Por su parte, $\mathrm{O}^{\prime}$ Neil y su equipo reportaron mejor función urinaria en ese mismo grupo de pacientes, ${ }^{10}$ diferente a nuestro estudio, donde la recuperación de la continencia en sujetos con prostatectomía radical abierta o laparoscópica asistida por robot fue similar a los 3 (53 vs 57\%; $\mathrm{p}=0.7$ ), 6 (88 vs 76\%; $\mathrm{p}=0.18$ ) y 12 meses ( 94 vs
$100 \% ; p=0.36$ ), datos que coinciden con la investigación de Jackson y colaboradores, quienes tampoco encontraron diferencias entre ambos abordajes (41.5 vs 48\%; $\mathrm{p}=0.72$ ). En cuanto a la potencia o desempeño sexual, Ficarra y sus coautores señalan mejor recuperación a 12 meses en pacientes con prostatectomía radical laparoscópica asistida por robot $(p=0.002),{ }^{14}$ similar a lo reportado por $\mathrm{O}^{\prime}$ Neil. Nuestros resultados mostraron mejor desempeño sexual a los 12 meses en los pacientes con prostatectomía radical laparoscópica asistida por robot (57 vs 36\%; $p=0.17$ ), aunque sin significación estadística, quizá porque los pacientes evaluados a los 12 meses con prostatectomía asistida por robot fueron menos respecto de los evaluados con prostatectomía radical abierta, debido al seguimiento más largo con esta última técnica.

El metanálisis de Leow y colaboradores reporta mayor costo de la prostatectomía radical laparoscópica asistida por robot vs prostatectomía radical abierta; sin embargo, se compensan por el número de prostatectomías robóticas realizadas en Estados Unidos. ${ }^{6}$ En nuestro hospital, este parámetro debe evaluarse a futuro.

Limitaciones de la investigación: se trata de un estudio retrospectivo, con un número reducido de pacientes, comparado con grandes series, y el tiempo de seguimiento con ambas opciones fue diferente; sin embargo, es importante reportar los resultados iniciales de nuestra experiencia.

\section{CONCLUSIONES}

Aunque la curva de aprendizaje de la prostatectomía radical laparoscópica asistida por robot se está forjando y la de prostatectomía radical abierta está consolidada, realizar un primer estudio comparativo entre ambos abordajes confirma las ventajas en cuanto a sangrado transoperatorio y transfusión de la primera técnica; 
además, el resto de los parámetros evaluados fueron similares, pero no inferiores, a la prostatectomía radical abierta. Un factor que influyó en la tasa elevada de márgenes positivos en la prostatectomía radical laparoscópica asistida por robot fue que se encontraron estadios más avanzados ( $\geq$ pT3) en pacientes en quienes se eligió esta opción de tratamiento. Con el paso del tiempo y al dominar el procedimiento se espera disminuir las complicaciones posquirúrgicas, estancia hospitalaria, márgenes positivos y mejorar la continencia urinaria y el desempeño sexual, como se ha reportado en los estudios internacionales. Conocer estos resultados iniciales permitirá considerarlos como referencia para realizar estudios comparativos a futuro y de forma prospectiva.

\section{Conflictos de interés}

Los autores declaran no tener conflictos de interés.

\section{Financiamiento}

No se recibió ningún recurso para financiar el estudio.

\section{REFERENCIAS}

1. Gandaglia G, et al. Comparative effectiveness of robotassisted and open radical prostatectomy in the postdissemination era. J Clin Oncol 2014;32:1419-1426.

2. Su LM, Gilbert SM, Smith JA. Laparoscopic and robotic-assisted radical prostatectomy and pelvic lymphadenectomy. En: Wein AJ, Kavoussi LR. Campbell-Walsh Urology. 11ㄹ ed. New York: McGraw Hill, 2015;2663-2684.

3. Lowrance W, Eastham J, Savage C, Maschino A, Laudone $V$, Dechet $C$, et al. Contemporary open and robotic radical prostatectomy practice patterns among urologists in the United States. J Urol 2012;187:2087-2093.
4. Schaeffer E, Partin A, Leport H. Open Radical prostatectomy. En: Wein AJ, Kavoussi LR. Campbell-Walsh Urology. 11a ed. New York: McGraw Hill, 2015;2641-2662.

5. Novara G, Ficarra V, Rosen RC, Artibani W, Costello A, Eastham JA, et al. Systematic review and meta-analysis of perioperative outcomes and complications after robotassisted radical prostatectomy. Eur Urol 2012;62:431-452.

6. Leow JJ, Chang SL, Meyer CP, Wang Y, Hanske J, Sammon $J D$, et al. Robot-assited versus open radical prostatectomy: a contemporary analysis of an all-payer discharge database. Eur Urol 2016;1:1-9.

7. Hu J, Gandaglia $G$, Karakiewicz $P$, Nguyen $P$, TrinH $Q, A b-$ dollah $F$, et al. Comparative Effectiveness of robot-assited versus open radical prostatectomy cancer control. Eur Urol 2014;66:666-672.

8. Pearce SM, Pariser JJ, Karrison T, Patel SG, Eggener SE. Comparison of perioperative and early oncologic outcomes between open and robotic assisted laparoscopic prostatectomy in a contemporary population based cohort. J Urol 2016;196:1-6.

9. Ficarra V, Novara G, Rosen RC, Artibani W, Carroll PR, Costello $A$, et al. Systematic review and meta-analysis of studies reporting urinary continence recovery after robotassisted radical prostatectomy. Eur Urol 2012;62:405-417.

10. O'Neil B, Koyama T, Alvarez J, Conwill R, Albertsen P, Cooperberg MR, et al. The comparative harms of open and robotic prostatectomy in population based samples. J Urol 2016;195:321-329.

11. Novara G, Ficarra V, Mocellin S, Ahlering TE, Carrol PR, Graefen $\mathrm{M}$, et al. Systematic review and meta-analysis of studies reporting oncologic outcome after robot-assisted radical prostatectomy. Eur Urol 2012;62: 382-404.

12. Mottet N, Bellmunt J, Briers E, Van Den Berg RCN, Bolla M, Van Casteren NJ, et al. Guidelines on Prostate Cancer. European Association of Urology. 2015:1-137.

13. Patel VR, Sivaraman A, Coelho RF, Chauhan S, Palmer KJ, Orvieto MA, et al. Pentafecta: A new concept for reporting outcomes of robot-assisted laparoscopic radical prostatectomy. Eur Urol 2011;59:702-707.

14. Ficarra V, Novara G, Ahlering TE, Costello A, Eastham JA, Graefen $M$, et al. Systematic review and meta-analysis of studies reporting potency rates after robot-assisted radical prostatectomy. Eur Urol 2012;62:418-430.

15. Jackson M, Bellas N, Siegrist T, Haddock P, Staff I, Laudone $V$, et al. Experienced open vs early robotic-assisted laparoscopic radical prostatectomy: a 10-year prospective and retrospective comparison. Urology 2016;91:111-118. 